\title{
Photopolymer-Based Stratified Volume Holographic Optical Elements
}

Gregory P. Nordin

nordin@byu.edu

A. R. Tanguay

Follow this and additional works at: https://scholarsarchive.byu.edu/facpub

Part of the Electrical and Computer Engineering Commons

\section{Original Publication Citation}

G. P. Nordin and A. R. Tanguay, Jr."Photopolymer-Based Stratified Volume Holographic Optical Elements," Opt. Lett. 17(23), pp. 179-1711 (1992)

\section{BYU ScholarsArchive Citation}

Nordin, Gregory P. and Tanguay, A. R., "Photopolymer-Based Stratified Volume Holographic Optical Elements" (1992). Faculty Publications. 711.

https://scholarsarchive.byu.edu/facpub/711

This Peer-Reviewed Article is brought to you for free and open access by BYU ScholarsArchive. It has been accepted for inclusion in Faculty Publications by an authorized administrator of BYU ScholarsArchive. For more information, please contact ellen_amatangelo@byu.edu. 


\title{
Photopolymer-based stratified volume holographic optical elements
}

\author{
Gregory P. Nordin* and Armand R. Tanguay, Jr. \\ Optical Materials and Devices Laboratory, Center for Photonic Technology, and National Center for Integrated Photonic Technology, \\ University of Southern California, Los Angeles, California 90089-0483
}

Received June 12, 1992

\begin{abstract}
We report the implementation of stratified volume holographic optical elements (SVHOE's) using DuPont's Omnidex holographic photopolymer material and an in situ exposure technique for simultaneous multilayer grating recording. Experimental measurement of the +1 -order angular sensitivity of a seven-layer SVHOE structure shows remarkable agreement with both theory and numerical simulation for incidence angles near the Bragg angle. For SVHOE's having modulation layers that individually operate in the Bragg or transition diffraction regimes, the envelope of the SVHOE angular sensitivity is experimentally shown to approximate closely that of a single modulation layer.
\end{abstract}

Stratified volume holographic optical elements (SVHOE's) are a recently proposed class of novel diffraction structure in which multiple layers of a thin holographic material are interleaved with optically homogeneous buffer layers. ${ }^{1-3}$ These structures exhibit unique diffraction properties with potential applications in areas such as optical array generation, ${ }^{1-3}$ wavelength notch filtering, and grating spatial frequency filtering., ${ }^{2,3}$ In addition, SVHOE's can be designed to emulate the diffraction properties of conventional volume holographic optical elements. ${ }^{1,3}$ As such, the SVHOE concept allows holographic media that are currently available only in thin-film form to be used in certain applications that require thick holographic optical elements. In this Letter we report the fabrication of photopolymerbased SVHOE structures using DuPont's Omnidex thin-film holographic material, ${ }^{4,5}$ which allows for simultaneous holographic recording in all the modulation layers.

The periodic angular sensitivity characteristic of ;VHOE diffraction behavior was first described in ief. 1 , in which both theoretical calculations and $n$ experimental demonstration were reported. The SVHOE used in the experimental measurement was constructed by stacking photoresist layers (each on its own substrate) in which identical diffraction gratings had previously been recorded. ${ }^{1}$ In SVHOE's fabricated with such prerecorded gratings, alignment of the grating fringes in the modulation layers is naturally an important issue, particularly when many layers are involved. The photopolymerbased SVHOE's described herein were exposed to grating recording beams after assembly of the structures. This in situ exposure technique provides for autoalignment of the gratings from layer to layer. Use of the DuPont photopolymer material (which is a permanent holographic medium) is especially convenient in this context because it requires no postexposure wet chemical fixing process (such as that required for both dichromated gelatin and Polaroid's
DMP-128 material). Instead, fixing is accomplished by exposure to a uniform beam at the wavelength of the grating recording beam or by exposure to ultraviolet illumination.

DuPont's holographic photopolymer material is available in formulations optimized for either transmission or reflection holograms and can contain dye sensitizers that cover several wavelength ranges in the visible spectrum. ${ }^{5}$ The blue-green-sensitized transmission photopolymer HRF-150 is available in several forms, the most common of which is a $38-\mu \mathrm{m}$-thick film on a flexible Mylar backing.

The modulation layers in our SVHOE structures were spin coated from a solution of HRF-150 photopolymer. Separate photopolymer and bluegreen dye solutions from DuPont were used to obtain custom photopolymer/dye concentrations. The composite solution used for fabrication of the seven-layer SVHOE illustrated in Fig. 1 consisted of a 1:110 ratio of dye solution to $15 \%$ photopolymer solution by weight. Thin films were formed on commercially available glass coverslips by spin coating at $3000 \mathrm{rpm}$ for $2 \mathrm{~min}$. The relatively long spin duration was used to aid in drying the films. The resultant photopolymer films were $1.8 \mu \mathrm{m}$ thick and had an absorption coefficient of $140 \mathrm{~cm}^{-1}$ at $488 \mathrm{~nm}$ (unexposed).

Because the photopolymer is tacky after spinning, the photopolymer-coated coverslips can be directly attached to a flat surface by pressing the film side of the coverslip onto the surface. In our case, the first photopolymer-coated coverslip was mounted directly onto a glass slide, and each subsequently coated coverslip was mounted onto the back surface of the preceding coverslip. The combined thickness of a coverslip and photopolymer layer was $150 \mu \mathrm{m}$.

For the particular SVHOE shown in Fig. 1, the number of modulation layers was limited to seven in order to minimize grating strength nonuniformities in the photopolymer layers that result from absorption in previous layers. From the parameters given 


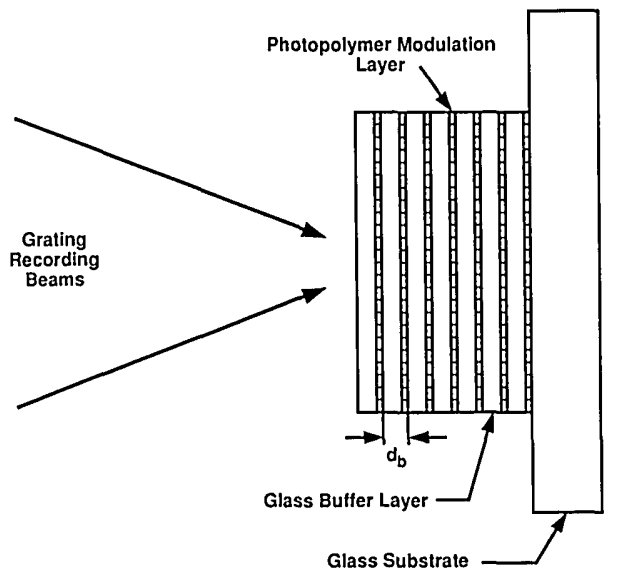

Fig. 1. Photopolymer-based SVHOE fabricated with glass substrates and exposed by using an in situ exposure technique.

above, we estimate a $2.5 \%$ absorption loss at each photopolymer layer at the beginning of an exposure. During the exposure process, the dye sensitizer is bleached in each layer, and absorption consequently decreases. To further ensure uniform grating strengths in the photopolymer layers, increased exposures of approximately $2 \mathrm{~J} / \mathrm{cm}^{2}$ were used (the diffraction efficiency typically saturated at exposures of between 90 and $140 \mathrm{~mJ} / \mathrm{cm}^{2}$ for the structures that we fabricated). In all cases, the intensity of each 488-nm recording beam was between 110 and $130 \mathrm{~mW} / \mathrm{cm}^{2}$, with both recording beams having the same intensities in order to achieve near-unity modulation depths.

Collimated recording beams with a $14.5^{\circ}$ angular separation were used to expose the seven-layer SVHOE shown in Fig. 1 . The resultant $1.93-\mu \mathrm{m}$ period of the grating written in the photopolymer layers was large enough relative to the individual layer thicknesses to ensure that each layer individually operated in the Raman-Nath diffraction regime.

The angular sensitivity of the seven-layer SVHOE +1 order was measured by using a $\mathrm{He}-\mathrm{Ne}$ laser beam at a wavelength of $632.8 \mathrm{~nm}$. The +1 -order diffraction efficiency is shown as a function of the incidence angle of the readout beam in Fig. 2. The diffraction efficiency is defined as the ratio of the power diffracted into the +1 order to the power in the incident readout beam. The angular range shown in Fig. 2 includes the diffraction peak at Bragg incidence $\left(9.44^{\circ}\right.$ ) and two neighboring peaks. The angular separation of the peaks is $1.13^{\circ}$, which agrees well with the calculated separation of $1.12^{\circ}$ (given in turn by $n \Lambda / d_{b}$, in which $n$ is the glass refractive index, $\Lambda$ is the grating period, and $d_{b}$ is the buffer layer thickness). 1,3

The refractive-index modulation in the photopolymer layers can be estimated from the peak diffraction efficiency of $5.4 \%$. Extensive numerical simulation ${ }^{3}$ shows that the relationship between the peak diffraction efficiency of an SVHOE and the refractiveindex modulation in the modulation layers is well approximated by the usual expression for a conventional Bragg grating, ${ }^{6} \sin ^{2}(\pi \Delta n d / \lambda)$, in which $\Delta n$ is the amplitude of the refractive-index modulation, $d$ is the total thickness of the modulation layers, and $\lambda$ is the wavelength of the readout beam. The refractive-index modulation calculated from this expression is $3.7 \times 10^{-3}$, which is approximately a factor of 2 less than that reported in Ref. 5 (for a corresponding grating period of $1.0 \mu \mathrm{m}$ ). This is consistent with our experimental observation that the refractive-index modulation recorded in the photopolymer exhibits a strong dependence on the grating spatial frequency.

With the use of this value for the refractive-index modulation, the angular sensitivity of the seven-layer SVHOE was simulated by using the optical beampropagation method ${ }^{1,7}$ (BPM). The result is shown as the dashed curve in Fig. 2. Note the remarkable agreement between numerical simulation and experimental measurement for both the main peaks and the sidelobes over the angular range shown in the figure. Comparison of the measured peak diffraction efficiency at Bragg incidence with that obtained by direct numerical simulation shows excellent agreement, thus validating the estimate of the refractiveindex modulation obtained above by applying the traditional relationship applicable for a conventional bulk grating.

For incidence angles outside of the angular range shown in Fig. 2, the agreement between experiment and numerical simulation decreases, in that the experimentally measured diffraction peaks assume a double-lobed structure and exhibit a larger angular separation than the BPM simulations predict. The angular locations of the peaks can be accurately predicted by considering the relative phases of the zero and +1 orders as they propagate from layer to layer ${ }^{3}$ without making the small-angle approximation that is incorporated in the BPM simulations.

After measurement of its angular sensitivity, the seven-layer SVHOE was disassembled layer by layer, and the angular sensitivity was remeasured after each photopolymer-coated coverslip was removed. Measurements for six, four, and two layers are shown in Fig. 3. Note that as the total device thickness decreases, the angular width of the diffraction peaks increases as expected. In addition, the sidelobe

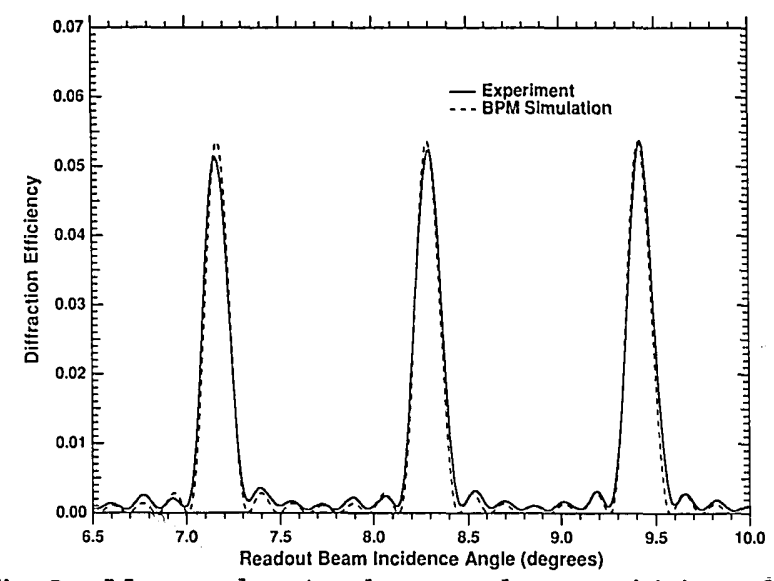

Fig. 2. Measured +1-order angular sensitivity of a seven-layer photopolymer-based SVHOE compared with numerical simulations based on experimental parameters. 


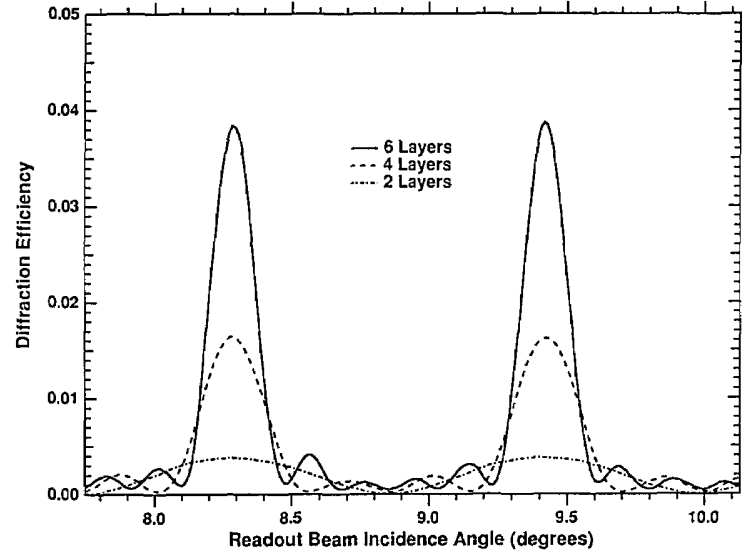

Fig. 3. Measured +1-order angular sensitivity of the same SVHOE as in Fig. 2, after successive removal of photopolymer-coated glass layers.

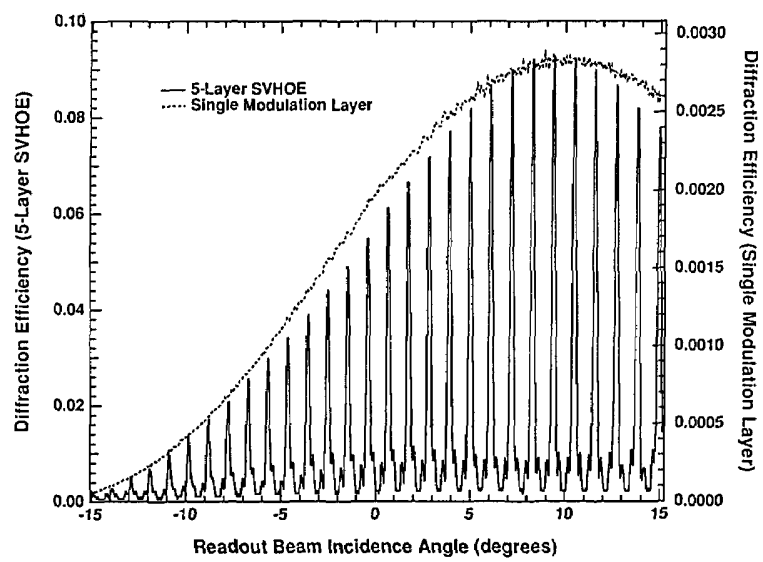

Fig. 4. Angular sensitivities of an identically exposed five-layer SVHOE structure and a single-layer photopolymer film. The single-layer photopolymer film thickness is the same as that of each individual photopolymer layer within the SVHOE.

structure is exactly as predicted in Refs. 1 and 3; i.e., an SVHOE having $N$ modulation layers has $N-$ 2 sidelobes between each pair of adjacent periodic diffraction peaks.

Not all thin-film photosensitive materials will be thin enough to yield strictly Raman-Nath diffraction behavior within each modulation layer under all possible conditions. When such modulation layers individually exhibit $\mathrm{Bragg}^{8}$ or transition regime (i.e., neither Bragg nor Raman-Nath) diffraction behavior, the uniform periodic angular sensitivity of an SVHOE is modified by an envelope function that should be equal to the angular sensitivity of a single modulation layer according to Ref. 1 . This behavior was experimentally verified as follows. A fivelayer SVHOE was constructed as described above, except that the thin-film photopolymer layers were spun from a $20 \%$ photopolymer solution at $2000 \mathrm{rpm}$, resulting in films that were $4.6 \mu \mathrm{m}$ thick. After assembly, a $1.93-\mu \mathrm{m}$ period grating was recorded in the structure. The angular sensitivity of the +1 order of the five-layer SVHOE is shown in Fig. 4, along with the angular sensitivity of a single $4.6-\mu \mathrm{m}$ thick photopolymer film exposed under identical conditions. Note that the angular sensitivity of the single photopolymer film does, in fact, closely approximate the envelope of the five-layer SVHOE diffraction response, with the exception that the former is slightly broader. The difference may be due to nonuniform grating amplitudes within each SVHOE photopolymer layer, unequal layer-to-layer grating strengths, variations in the buffer layer thicknesses, or some combination thereof.

In summary, we have demonstrated the fabrication of SVHOE structures using a photopolymer holographic material that allows for in situ multilayer grating exposure. For SVHOE's with modulation layers that individually operate in the Raman-Nath regime, experimental measurements of the +1-order angular sensitivity were found to be in excellent agreement with both theoretical predictions and numerical simulations for angles near Bragg incidence. For SVHOE's with thick modulation layers that operate in the transition or Bragg regimes, the angular sensitivity of a single modulation layer was experimentally demonstrated to determine the envelope of the periodic +1-order diffraction peaks, in agreement with a previous theoretical prediction.

This research was supported in part by the Defense Advanced Research Projects Agency through the U.S. Air Force Office of Scientific Research (National Center for Integrated Photonic Technology). G. Nordin gratefully acknowledges support from a Hughes Doctoral Fellowship and a Center for Photonic Technology Graduate Prize Fellowship at the University of Southern California. We are pleased to express our appreciation to E. I. du Pont de Nemours and Company, Inc., for making the photopolymer material available to us.

*Present address, Department of Electrical and Computer Engineering, The University of Alabama in Huntsville, Huntsville, Alabama 35899.

\section{References}

1. R. V. Johnson and A. R. Tanguay, Jr., Opt. Lett. 13, 189 (1988).

2. G. P. Nordin, R. V. Johnson, and A. R. Tanguay, Jr., Annual Meeting, Vol. 11 of 1988 OSA Technical Digest Series (Optical Society of America, Washington, D.C., 1988), p. 106.

3. G. P. Nordin, R. V. Johnson, and A. R. Tanguay, Jr., "Diffraction properties of stratified volume holographic optical elements," J. Opt. Soc. Am. A (to be published).

4. W. K. Smothers, B. M. Monroe, A. M. Weber, and D. E. Keys, Proc. Soc. Photo-Opt. Instrum. Eng. 1212, 20 (1990).

5. A. M. Weber, W. K. Smothers, T. J. Trout, and D. J. Mickish, Proc. Soc. Photo-Opt. Instrum. Eng. 1212, 30 (1990).

6. H. Kogelnik, Bell Syst. Tech. J. 48, 2909 (1969).

7. J. A. Fleck, J. R. Morris, and M. D. Feit, Appl. Phys. 10, 129 (1976).

8. A. P. Yakimovich, Opt. Spectrosc. (USSR) 49, 85 (1980). 